EL USO DE REDES SOCIALES EN LAS MICROEMPRESAS PANIFICADORAS DE TABASCO.

\title{
EL USO DE REDES SOCIALES EN LAS MICROEMPRESAS PANIFICADORAS DE TABASCO
}

\section{THE USE OF SOCIAL NETWORKS IN MICRO ENTERPRISES OF BAKERY IN TABASCO}

Miguel Ángel Valenzuela Ramos*, María del Carmen Navarrete Torres**

\begin{abstract}
*Pasante de la Licenciatura en Mercadotecnia. Universidad Juárez Autónoma de Tabasco. División Académica de Ciencias Económico Administrativas. ORCID: https://orcid.org/0000-0002-9430-5117.

**Doctora en Educación. Universidad Juárez Autónoma de Tabasco. División Académica de Ciencias Económico Administrativas. Email: mallynav@yahoo.com.mx. ORCID: https://orcid.org/0000-0003-4633-
\end{abstract} 0323.

Dirección para recibir correspondencia: m_valenzuela3@outlook.com 
EL USO DE REDES SOCIALES EN LAS MICROEMPRESAS PANIFICADORAS DE TABASCO.

\section{RESUMEN}

En la actualidad, las redes sociales han cobrado gran relevancia en el sector económico del país, constituyéndose como un elemento de gran importancia para el desarrollo y posicionamiento de cualquier empresa. La innovación de los sistemas de producción y distribución, se caracteriza por la adopción de las herramientas digitales contemporáneas.

OBJETIVO: Determinar el nivel de uso que las microempresas dedicadas a la producción de pan en el estado de Tabasco, específicamente en la ciudad de Villahermosa les dan a dichas herramientas para aumentar sus niveles de venta.

MATERIAL Y MÉTODO: Se utilizó una metodología no experimental mixta con un diseño transeccional.

RESULTADOS: En la investigación se encontró que más del 50\% de las microempresas panaderas en Villahermosa no usan redes sociales. Así mismo se comprobó que únicamente un $20 \%$ de los empresarios cree tener conocimiento referente a las ventajas del uso de herramientas digitales.

CONCLUSIÓN: Derivado del análisis podemos determinar que los comercios dedicados al expendio de pan en el estado presentan niveles bajos en relación al uso de medios digitales, por lo que se propone que los empresarios deberán capacitarse para el uso de dichas herramientas a fin de tener una mayor ventaja competitiva.

PALABRAS CLAVE: Microempresas. Panaderías. Redes Sociales.

\section{ABSTRACT}

Nowadays, the social networks have gained great relevance in the economic sector of the country, becoming an element of great importance for the development and positioning of any company. The innovation of production and distribution systems is characterized by the adoption of contemporary digital tools. 
EL USO DE REDES SOCIALES EN LAS MICROEMPRESAS PANIFICADORAS DE TABASCO.

OBJECTIVE: To determine the level of use that microenterprises devoted to bread production in the state of Tabasco, specifically in the city of Villahermosa, give to these digital tools to increase their sales levels.

MATERIAL AND METHOD: A mixed non-experimental methodology with a transectional design was used.

RESULTS: The research found that more than $50 \%$ of the bakery microenterprises in Villahermosa do not use social networks. Likewise, it was found that only $20 \%$ of the entrepreneurs believe to have knowledge regarding the advantages of using digital tools.

CONCLUSION: Derived from the analysis, we can determine that the businesses dedicated to the sale of bread in the state have low levels in relation to the use of digital media, so it is proposed that entrepreneurs should be trained to use such tools in order to have a greater competitive advantage.

KEYWORDS: Microenterprise. Bakery businesses. Social networks.

\section{INTRODUCCIÓN}

De acuerdo con Forbes (2010), la gastronomía mexicana ha sido declarada Patrimonio Cultural Inmaterial de la Humanidad por la Organización de las Naciones Unidas para la Educación, la Ciencia y la Cultura. Como resultado, esta se considera un componente destacado de la identidad nacional.

En México, uno de los alimentos de mayor consumo diario, como complemento de todo tipo de comidas es el pan. Trujillo (2009) señala que no hay otro producto alimenticio que posea una historia tan extensa y repleta de simbología como este. A través de los siglos, la falta de pan se ha considerado como un sinónimo de insuficiencia económica y austeridad.

Según datos de la Cámara Nacional de la Industria Panificadora y Similares de México (2016) la panadería constituye la tercera industria generadora de empleo en México, tan solo por debajo VALENZUELA-RAMOS M. Á., NAVARRETE-TORRES M. DEL C. 
EL USO DE REDES SOCIALES EN LAS MICROEMPRESAS PANIFICADORAS DE TABASCO.

de la industria de la construcción y la automotriz con más de 2 millones de empleos: 500 mil directos y 1 millón 500 mil indirectos.

En Tabasco existen alrededor de 9,500 unidades económicas consideradas microempresas comercializadoras de alimentos y bebidas, 572 de estas entran en el rubro de la Panificación (INEGI, 2016).

En el Sur del país, específicamente en Tabasco, hay una gran cantidad de productos de panadería que destacan por su excelente sabor y las historias que rodean la fabricación de cada pieza. Ejemplo de esto son: el pan de yuca, pan de chaya, pan de plátano, el pan Tabasco, las galletas de sal, los kekes, el tacón, el marido, el cocotazo, así como el "riñón" relleno de queso y membrillo tan característico del municipio de Centla (Gobierno de Tabasco, 2016; Arecetas, 2019).

Las microempresas conforman la columna vertebral del aparato económico y productivo en México; sin embargo, estas enfrentan una limitada permanencia en el mercado y una minúscula participación en los mercados internacionales, por lo que requieren evolucionar para responder positivamente a las demandas y desafíos del mercado global. Dichos desafíos incluyen la incorporación de nuevas tecnologías y la reingeniería de los procesos de producción, distribución y comercialización que caracterizan los modelos de venta contemporáneos (Sánchez y Valdés 2011).

En 1995, Goody afirmó que la distribución y consumo de los alimentos incrementa a través del tiempo, con base en el crecimiento de la población mundial, sus gustos y preferencias. No obstante, la falta de información y el desconocimiento de los beneficios inherentes al uso de la tecnología disponible, puede resultar en una desventaja para algunas empresas que no pueden ir a la par de la creciente tendencia relacionada con los perfiles digitales y los medios de comunicación no tradicionales.

Estudios realizados por Williams, Crittenden, Keo, \& McCarty (2012) y Xiang \& Gretzel (2010) señalan que en esta década los medios digitales han afectado el comportamiento de compra del consumidor. A su vez, Fayol (1987) infiere que para lograr la destreza comercial y tomar decisiones empresariales con astucia, se necesita un profundo conocimiento del mercado y de la fuerza de los competidores. 
EL USO DE REDES SOCIALES EN LAS MICROEMPRESAS PANIFICADORAS DE TABASCO.

En este caso, los cada vez más novedosos canales de distribución, la exposición multimedios, así como la presencia en línea son algunas de las estrategias y recursos utilizados por las grandes empresas panaderas y diversas cadenas de supermercados, que tienen como consecuencia la alta exposición de sus productos y una perspectiva más amplia del gusto y la opinión de sus consumidores.

Así pues, es pertinente el análisis de los micro negocios del sector panadero ubicados en Villahermosa, esto con la finalidad de identificar, aquellas micro empresas que hacen uso de algunas de las herramientas del marketing digital.

\section{Marco teórico}

Se llama marketing al conjunto de esfuerzos encaminados a detectar y satisfacer las necesidades de un cliente. Dichas necesidades pueden ser de carácter básico tales como comida y vestido, o incluso aquellas que tienen como resultado la realización y el reconocimiento personal. Los esfuerzos inician con la concepción de una idea que permita la creación de un producto o servicio que satisfaga las necesidades de un individuo o de una comunidad. Para esto se tendrán en cuenta herramientas como la investigación de mercados, que permitan el establecimiento de características específicas, un precio ideal, los canales de distribución adecuados, así como un pertinente plan de promoción de ventas (Cervantes, 2016).

La American Marketing Association (2013) dice que: El marketing es la actividad realizada por un conjunto de instituciones y procesos para crear, comunicar, entregar e intercambiar ofertas que tienen valor para los clientes, socios y la sociedad en general.

Esta disciplina se ha abierto paso hasta convertirse en un elemento inherente al gen de cualquier empresa que compita en el mercado para ofrecer a los clientes el valor de sus productos o servicios. Prácticamente todas las empresas usan estrategias de mercadotecnia, en algunos casos sin percatarse de ello, en otros, dichas estrategias son realizadas de forma rudimentaria (Marketing FCA, 2009).

El perfil de los consumidores de este siglo es por demás diferente al del siglo pasado. Hoy en día, las organizaciones se enfrentan a un cambio en las motivaciones de compra de los clientes, lo que resulta en variaciones de los hábitos de compra, así como en el uso y demanda de servicios 


\section{EL USO DE REDES SOCIALES EN LAS MICROEMPRESAS PANIFICADORAS DE TABASCO.}

y productos. El consumidor del siglo XXI tiene todo al alcance de su mano, a un clic de distancia, posee gran información de los avances tecnológicos, conoce con claridad sus derechos y obligaciones, así como las de su proveedor y está dispuesto a exigir el cumplimiento de los acuerdos realizados, y en caso de no quedar satisfecho con la experiencia ofrecida, analiza con rapidez un cambio de proveedor (Cuesta, 2010). Dichas razones exigen la evolución de los procesos de promoción e intercambio de bienes y servicios, así como de las estrategias en torno a las experiencias que las organizaciones ofrecen a este nuevo perfil de consumidores.

La popularización del internet, de la mano con la creación y mejora de las tecnologías de la información, han traído como resultado el incremento de la competitividad empresarial a una escala global. Esto a su vez, ha impulsado en las organizaciones el desarrollo de condiciones de comercio más justas y al alcance de todos (Cervantes, 2016).

Se enfrenta pues, la necesidad de crear estrategias de desarrollo que aseguren la distribución de productos y servicios, así como la proyección de estos bienes en una economía móvil (Ramírez, 2004). Estos esfuerzos se conceptualizan en el término marketing digital. De acuerdo con ElGohari (2010) este puede ser visto como una nueva filosofía basada en el marketing de bienes, servicios e ideas que usa el internet y otros medios electrónicos como principal vía de comercio. Según Smith and Chaffey (2005) Este tipo de marketing se enfoca en "alcanzar objetivos mediante la aplicación de tecnologías electrónicas".

Desde la perspectiva de Poblete (2013) es una disciplina que se fundamenta en el uso de medios tecnológicos con el fin de actualizar de forma permanente una base de datos que provea de eficiencia la comunicación directa con su mercado meta. En México es aún una ciencia en desarrollo y tiene como origen los cambios es la sociedad, tales como nuevos procesos de producción, así como las distintas plataformas que integran los medios de información, particularmente tras la aparición del internet como elemento de valor en la vida de los consumidores (Figueroa, 2015).

Hoy en día, existen diversas sub-herramientas en línea como: motores de búsqueda, páginas de internet empresarial, Search Engine Marketing, redes sociales, blogs, foros, e-mail marketing, publicidad display, marketing móvil, advergaming e inbound marketing (Santes, 2017). Tales herramientas se refieren de manera indistinta a las aplicaciones de la mercadotecnia tradicional, pero adaptadas al desarrollo tecnológico reciente (Gándara, 2018). 
EL USO DE REDES SOCIALES EN LAS MICROEMPRESAS PANIFICADORAS DE TABASCO.

El ritmo de trabajo de las empresas se encuentra en constante cambio, todo apunta hacia lo intangible (Flórez, 2012). Dichos cambios afectan la actitud y el comportamiento de los consumidores. Es así, que el universo portable permea cada vez más a la sociedad y origina modernos estilos de vida y nuevos hábitos de consumo (Andrade, 2016). Las cada vez más novedosas redes sociales, la inmediatez en los procesos y servicios, así como la posibilidad de analizar en tiempo real los resultados de la aplicación de las diversas estrategias de medios, tienen lugar en los sistemas tecnológicos de inteligencia (Agencia DM Marketing Digital, 2015).

El concepto de redes sociales es amplio, tanto como sus aplicaciones en la vida práctica de miles de usuarios; éstas herramientas tienen un alto nivel de importancia en el diseño e implementación de las estrategias de promoción, distribución y comercialización de cualquier empresa (Calderón, 2015). Por definición, éstas suelen relacionarse con los intercambios dinámicos de carácter comunicativo entre personas o grupos en contextos de diversa complejidad. Asimismo, busca identificar necesidades y problemáticas a través de un sistema abierto y en constante construcción involucrando un amplio espectro de recursos disponibles (Martínez, 2012).

De acuerdo con Hütt (2012) estas redes se constituyen por relaciones interpersonales, un rasgo completamente inherente a la naturaleza humana aplicado al entorno cambiante y diversificado que caracteriza la vida moderna llena de herramientas tecnológicas. Dichas herramientas son canales de propagación que involucran medios tradicionales y planos virtuales, dentro de los cuales estas redes son un elemento característico (Muñoz, 2016).

El surgimiento y propagación del uso de las redes sociales se deben en gran manera a los avances en el desarrollo de equipos de telefonía móvil, pues estos se encuentran a disposición directa de las masas para su uso y adaptación, el resultado de esto es la gestión de relaciones sociales a cualquier nivel (Aucay, 2017).

Cada aplicación tiene funciones que las distinguen unas de otras, y aun cuando todas buscan aumentar la eficiencia de la conexión entre dos entes, podemos diferenciarlas mediante su uso y aplicación (Aucay, 2017). A continuación, se presenta la tabla 1 con las principales herramientas y la descripción de cada una de ellas. 


\section{EL USO DE REDES SOCIALES EN LAS MICROEMPRESAS PANIFICADORAS DE TABASCO.}

\section{Tabla 1}

Tipos de redes

\begin{tabular}{|c|c|c|}
\hline Red Social & Tipo de Red Social & Funciónes \\
\hline Facebook & $\begin{array}{l}\text { Red social generalista; Puesto que albergan a } \\
\text { todo tipo de personas y admite todo tipo de } \\
\text { contenido } \\
\text { (Martos, 2010, p.73) }\end{array}$ & $\begin{array}{c}\text { "Las páginas profesionales de Facebook páginas } \\
\text { de fans o fan page permite a las empresas y } \\
\text { profesionales tener una presencia en Facebook } \\
\text { que es visible, no solo por los usuarios de } \\
\text { Facebook, sino por cualquier usuario de internet" } \\
\text { (Carballar, 2012, p. 93) }\end{array}$ \\
\hline Youtube & $\begin{array}{l}\text { Es una red social de contenidos, donde el } \\
\text { principal factor es el material que en esa red se } \\
\text { comparte } \\
\text { (Junta de castilla y León, 2012, p.8) }\end{array}$ & $\begin{array}{l}\text { "Utilizar YouTube en las estrategia de redes } \\
\text { sociales de su empresa, le permite diferenciarse } \\
\text { de sus competencia" (Mejía, 2013a, p.142) }\end{array}$ \\
\hline Twitter & $\begin{array}{l}\text { Es una red social con un servicio de } \\
\text { microblogging gratuito que permite a los } \\
\text { usuarios enviar y compartir entradas cortas, } \\
\text { denominadas "tweets", no mayores de } 140 \\
\text { caracteres. (Medina y Montjoy, 2015, p. 8) }\end{array}$ & $\begin{array}{c}\text { "Esta red social se ha convertido en un medio } \\
\text { social de interés para que las empresas puedan } \\
\text { comunicar determinadas ofertas, resolver las } \\
\text { dudas..." } \\
\text { (Gómez \& Suárez, 2012a, p.195) }\end{array}$ \\
\hline Linkedln & $\begin{array}{l}\text { Es una red social profesional } \\
\text { (Hütt, 2012, p.123) }\end{array}$ & $\begin{array}{l}\text { Linkedln, "se ha convertido en un soporte } \\
\text { especialmente atractivo para la inclusión de } \\
\text { anuncios y mensajes publicitarios que se pueden } \\
\text { dirigir a un público objetivo muy bien definido" } \\
\text { (Gómez \& Suárez, 2012a, p.195) }\end{array}$ \\
\hline Instagram & $\begin{array}{c}\text { Es una red social basada en compartir } \\
\text { elementos multimedia (Estrade, Soro \& } \\
\text { Hernández, 2013, p.131) }\end{array}$ & $\begin{array}{c}\text { En Instagram, "Las imágenes para compartir se } \\
\text { pueden modificar a través de una serie de filtros } \\
\text { que se han ido sofisticando a lo largo de las } \\
\text { sucesivas versiones del rograma" (Mejía, 2013d, } \\
\text { p.152) }\end{array}$ \\
\hline
\end{tabular}

Fuente: Elaboración propia con datos de Martos (2010), Medina y Montjoy (2015), Hütt (2012), Estrade, Soro \& Hernández (2013), Carballar (2012), Mejía (2013), Gómez \& Suárez (2012).

\section{Ventajas del marketing digital}

El traslado de lo analógico al ciberespacio requiere de un cambio de mentalidad, así como de un giro en el uso y diseño de procesos y medios. Hoy se cuenta con posibilidades que antes eran inimaginables. Por ejemplo, hoy en día cualquier persona que ve un programa de televisión es capaz de interactuar a la distancia, a través de una aplicación móvil o en sus redes sociales; seguir tendencias, compartir sus gustos, opiniones o intereses. Incluso los consumidores pueden usar más de una plataforma al mismo tiempo (Razón y Palabra, 2010). 


\section{EL USO DE REDES SOCIALES EN LAS MICROEMPRESAS PANIFICADORAS DE TABASCO.}

Rotondaro (2002) afirma, que se necesita implementar un modelo de negocio que se adapte de mejor forma al contexto actual, uno que permita realizar mejoras de manera amplia a los servicios y productos ofertados. Mientras que Peng \& Trappey (2005) sugieren que hacer una adopción formal del internet y mostrar su presencia en dicha herramienta debería ser una prioridad para las organizaciones modernas.

El marketing interactivo ocupa entonces un lugar preponderante en la eficacia de la comunicación y el alcance de objetivos en cualquier empresa. Ahora es más evidente que una campaña de promoción tradicional recibe mucha menos atención que una campaña creativa de marketing digital (Mattiqleppaniemi, 2010). Diversos hallazgos señalan que la adopción de las estrategias ideales proveerá a las empresas ciertas ventajas que redundan en una posición de privilegio dentro de las ofertas del mercado. Solinam \& Youssef (2003) mencionan que el obtener una perspectiva completa sobre la situación de la industria, así como la posibilidad de obtener una manera más efectiva para conocer la situación del mercado meta son algunas de las ventajas más importantes.

Si bien es cierto, el marketing digital ofrece muchas ventajas a quienes ostenten su uso y aplicación como parte de un plan de mercadotecnia integral. Dentro de las principales ventajas se encuentran las diversas métricas que describen el éxito o fracaso de la aplicación, su capacidad de segmentación, la personalización y flexibilidad, lo que da lugar a la interactividad; tiene un sentido completamente global y contempla la idea de la viralidad de los contenidos (Observatorio Regional de la Sociedad de la Información de Castilla y León, 2012; Cuesta, 2010; Cervantes, 2016).

Actualmente, se considera a los micronegocios como el sector predominante en la economía de los países desarrollados, así como de los emergentes o en vías de desarrollo. Tal es el caso de México, en el que dichas empresas son altamente reconocidas por ser quienes contribuyen con la mayor cantidad de empleos a la economía nacional (Mead \& Liedholm, 1998).

De acuerdo con la descripción ofrecida en las reglas de operación del Fondo PyME, publicado en el Diario Oficial de la Federación (2013), se considera microempresa a una empresa de cualquier sector, que tenga como máximo un total de diez trabajadores y que tenga un rango de monto de ventas anuales de hasta 4 millones de pesos. 


\section{EL USO DE REDES SOCIALES EN LAS MICROEMPRESAS PANIFICADORAS DE TABASCO.}

Existen diferencias evidentes entre las microempresas y las empresas pequeñas o medianas. Dentro de las principales podemos referir la estructura de la organización con la que cuentan, así como una mayor disponibilidad de recursos, lo que de forma indirecta circunscribe su área de impacto de forma relevante (Maldonado, 2017).

Diversas investigaciones concluyen que más del 90\% de las empresas que existen en Latinoamérica tienen un modelo de negocio familiar, ya que son algunos de estos miembros quienes dirigen, ejecutan o controlan las operaciones diarias (Borkowski, 2001; Carraher, 2005; Treviño-Rodríguez, 2009).

En un estudio auspiciado por el Banco Interamericano de Desarrollo (1997) se concluyó que las empresas familiares no solo ejercen una influencia directa en la economía, sino también en aspectos socioculturales de toda América Latina y gran parte del mundo (Santos y Guzmán, 2017). Otras investigaciones indican que estos entes ejercen un alto impacto positivo en la disminución de la pobreza, producto de los cambios en la sinergia económica de los lugares en los que se establecen (Santos y Guzmán, 2017; Agyapong, 2010; Gebremariam, Gebremedhim \& Jackson, 2004). Todas las empresas crean nuevas oportunidades de empleo, permiten el crecimiento y desarrollo de las entidades, también favorecen la creación de vínculos y lazos de carácter social que benefician a la comunidad de la que forman parte (Coraggio, 2003).

\section{Microempresas panaderas}

De acuerdo con Encuesta Nacional sobre Productividad y Competitividad de las Micro, Pequeñas y Medianas Empresas (INEGI, 2016) en Tabasco se ubican alrededor de 63696 unidades económicas, 23313 de estas en el municipio de Centro, y 9500 se encuentran dentro de la clasificación de micronegocios relacionadas con la producción y distribución de alimentos y bebidas. Existen pues, un total de 572 comercios en la entidad dentro rubro de la Panificación, mientras que Villahermosa cuenta con al menos 86 unidades en la misma clasificación.

Muchos de los microempresarios panaderos carecen de una formación administrativa que les permita tomar decisiones que aseguren el funcionamiento y desarrollo óptimo de las estrategias de crecimiento (Priego, 2013). La mayoría de estos toman decisiones con base en el instinto de imitación, ya que desconocen el uso de las herramientas administrativas tecnológicas y por lo tanto también las ventajas inherentes al correcto uso y aplicación de las mismas (Priego, 2013). 
EL USO DE REDES SOCIALES EN LAS MICROEMPRESAS PANIFICADORAS DE TABASCO.

\section{MATERIAL Y MÉTODO}

Se utilizó una metodología no experimental mixta con un diseño transeccional, dado que los datos se recolectaron en un tiempo específico y describen una situación de interés para conocer una variable, que en este caso son las herramientas del marketing digital utilizadas por las microempresas panaderas (Hernández, 2014).

Para la investigación, se realizaron 70 entrevistas a unidades económicas como muestra con un nivel de confianza del 95\%. El instrumento de investigación que se utilizó fue un cuestionario compuesto por 19 reactivos: cuatro de datos generales, cinco de carácter dicotómico y 10 de respuesta múltiple.

\section{RESULTADOS}

Se encontró que el 73\% de las microempresas dedicadas a la fabricación de pan, tienen una antigüedad superior a los ocho años, mientras que el 1\% tiene una antigüedad menor a los cuatro años (ver tabla 2).

\section{Tabla 2}

Antigüedad promedio de las microempresas panaderas

\begin{tabular}{lc}
\hline Tiempo de vida & Porcentaje \\
\hline $2-3$ años & $1 \%$ \\
$4-5$ años & $13 \%$ \\
$6-7$ años & $13 \%$ \\
8 años o más & $73 \%$ \\
\hline
\end{tabular}

Fuente: Elaboración propia.

Respecto al uso de las herramientas digitales, en la tabla 3 se muestra cómo el 51\% de las empresas encuestadas no emplean ninguna red social; mientras tanto, del 49\% restante que sí hace uso de redes sociales, el 79\% manifestó que las usan para recibir pedidos con anticipación y el $20 \%$ lo hace con fines publicitarios. 
EL USO DE REDES SOCIALES EN LAS MICROEMPRESAS PANIFICADORAS DE TABASCO.

Tabla 3

Uso de redes sociales

\begin{tabular}{lccc}
\hline Red social & Porcentaje de uso & Publicidad & Pedidos \\
\hline Facebook & $6 \%$ & & \\
Whatsapp & $43 \%$ & $20.59 \%$ & $79.40 \%$ \\
Ninguna & $51 \%$ & & \\
\hline
\end{tabular}

Fuente: Elaboración propia.

En lo que respecta al conocimiento de las redes sociales, se comprueba que solo el $20 \%$ de los empresarios cree tener conocimientos de las ventajas del uso de estas herramientas. Por otro lado, únicamente el 6\% de los encuestados afirmaron haber recibido alguna clase de capacitación sobre el tema (ver tabla 4).

Tabla 4

Conocimiento de las ventajas del uso de redes sociales

\begin{tabular}{lc}
\hline Si & No \\
\hline $\begin{array}{l}\text { Conocimiento de las } \\
\text { ventajas de las redes }\end{array}$ & $1 \%$ \\
$\begin{array}{l}\text { Capacitación para el uso de } \\
\text { redes }\end{array}$ & $13 \%$ \\
\hline
\end{tabular}

Fuente: Elaboración propia.

\section{CONCLUSIONES}

Los microempresarios locales dedicados a la elaboración de pan, saben de algunas de las ventajas de nuevas formas de publicidad para sus productos; sin embargo, desconocen el uso de herramientas digitales y su aplicación.

El no saber cómo utilizar adecuadamente las redes sociales para las microempresas, los coloca en desventaja y pierden la oportunidad de un mejor posicionamiento en el mercado y alcanzar mayores niveles de ventas. 
EL USO DE REDES SOCIALES EN LAS MICROEMPRESAS PANIFICADORAS DE TABASCO.

Los medios tradicionales siguen ocupando un lugar preponderante en la mente de los empresarios a la hora de anunciar sus ofertas o productos de temporada, otra gran parte de ellos aún considera que no hay mejor publicidad que la de boca en boca. Este principio, ha sido utilizado por diversas empresas para el desarrollo de software especializado, herramientas fáciles de mostrar y enseñar a este nicho de mercado que se aferra a elementos del comercio tradicional. Tal es el caso de Google Maps y Marketplace de Facebook que, a pesar de estar sujetas a un alto grado de subjetividad, les permite a los usuarios calificar de forma positiva o negativa lugares públicos a través de reseñas.

Por lo antes mencionado, se propone que los empresarios a fin de tener una ventaja competitiva mayor deberán capacitarse para el uso de las redes sociales y diversas herramientas digitales, ya que el constante avance de la tecnología acrecienta la brecha entre sus productos y los clientes potenciales. Muchas de estas empresas, se encuentran afiliadas a la Cámara Nacional de la Industria Panificadora y Similares, o a la Cámara Nacional de Comercio, Servicios y Turismo, por lo que se recomienda soliciten programas de capacitación para el uso y aplicación de las herramientas tecnológicas disponibles.

Si bien, los micro negocios de panaderos enfrentan serios problemas ante la llegada de grandes competidores y cadenas de supermercados, el uso de las herramientas digitales tiene el potencial de exponer el talento de las familias que generación a generación imprimen un sello de calidez y orgullo a los productos de la panadería típica del Sur de México.

\section{REFERENCIAS BIBLIOGRÁFICAS}

Agencia DM Marketing Digital. (2015). DM marketing digital. Recuperado el 20 de mayo de 2015, de http://www.mdmarketingdigital.com/que-es-el-marketing-digital.php

Agyapong, D. (2010). Micro, small and medium enterprises activities. Income level and poverty reduction in Ghana.

American Marketing Association. (2014). Definition of marketing. Recuperado el 27 de 2 de 2015, de AMA: https://www.ama.org/AboutAMA/Pages/Definition-of-Marketing.aspx 
EL USO DE REDES SOCIALES EN LAS MICROEMPRESAS PANIFICADORAS DE TABASCO.

Andrade, D. (2016). Estrategias de marketing digital en la promoción de Marca Ciudad. Revista Escuela de Administración de Negocios (Enero-Junio). Recuperado el día 20 de enero de 2019. Disponible en: http://www.redalyc.org/articulo.oa?id=20645903005

Arecetas (2019). Gastronomía de Tabasco. Recuperado de https://www.arecetas.com/gastronomia-america-americadelnorte-mexico-tabasco.html

Aucay, P. y Herrera, P. (2017) Nivel de uso de las redes sociales en el proceso de comunicación en las MIPES de Cuenca. Universidad Politécnica Salesiana.

Banco Interamericano de Desarrollo. (1997). América Latina tras una década de reformas. Washington, DC.

Borkowski, M. (2001). Options for buying and selling a family business. Canadian Plastics.

Calderón, W.; Abad, Y.; León, B. y Núñez, N. (2015). Nivel de utilización de las redes sociales por las MYPIMES de la ciudad Piura. Congresso de Contabilidade. Universitat Do Valencia, España.

Cámara Nacional de la Industria Panificadora y Similares de México. (2016). Panorama actual de la industria panificadora en México. Recuperado de http://usapeec.org.mx/publicaciones/presentaciones/pdf/panorama_actual_de_la_industri a_panificadora_en_mexico_2016.pdf

Carballar, J. (2012). Social media marketing personal y profesional. México: Alfaomega.

Carraher, M. (2005). An examination of entrepreneurial orientation: A validation study in 68 countries in Africa, Asia, Europe, and North America. International Journal of Family Business.

Cervantes, T. (2016). Mercadotecnia digital para el posicionamiento de las marcas de mezcal.

Coraggio, J. (2003). La Economía Social como vía para otro desarrollo social. Argentina: Universidad Nacional General Sarmiento.

Cuesta, F. (2010). Marketing directo 2.0. Barcelona: Grupo Planeta.

Diario Oficial de la Federación (2013) REGLAS de Operación del Fondo de Apoyo para la Micro, Pequeña y Mediana Empresa (Fondo PyME) para el ejercicio fiscal 2013. Recuperado de: http://www.dof.gob.mx/nota_detalle.php?codigo=5289919\&fecha=28/02/2013 
EL USO DE REDES SOCIALES EN LAS MICROEMPRESAS PANIFICADORAS DE TABASCO.

El-Gohary, H. (2010) E-Marketing - A Literature Review from a Small Businesses perspective.

Estrade, J., Soro, D. \& Hernández, Á. (2013). Marketing Digital. Madrid: Ediciones Anaya.

Fayol, H. (1987). Administración industrial y general. Argentina: El Ateneo.

Figueroa, S. (2015). Estrategia de marketing digital en redes sociales para Pymes mexicanas: aplicación al Centro Universitario de América (CUAM) (Tesis de Posgrado), UNAM, México.

Forbes. (2016). Cómo llegó la gastronomía mexicana a ser patrimonio de la humanidad. Recuperado de https://www.forbes.com.mx/forbes-life/gastronomia-mexicana-patrimoniode-la-humanidad/

Flórez, C. (2012). Plataforma de innovación para el sector textil - confección. Medellín: (s.e.).

Gándara, M. (2018) La mercadotecnia digital como estrategia para fomentar e incrementar la movilidad.

Gebremariam, G., Gebremedhin, T. \& Jackson, R. (2004). The role of small business in economic growth and poverty alleviation in West Virginia: An Empirical Analysis.

Gobierno de Tabasco (2016). Gastronomía tabasqueña. Recuperado de https://web.archive.org/web/20110906015040/http://www.tabasco.gob.mx/estado/cultura _gastro.php

Gómez, Á. \& Suárez, C. (2012). Sistemas de información. Madrid: RA-MA Editorial.

Goody, J. (1995) Cocina, cuisine y clase: Estudio de sociología comparada. Barcelona: Editorial Gedisa.

Hernández, R., Fernández, C., Baptista, P. (2014). Metodología de la investigación. México: McGraw-Hill.

Hütt, H. (2012). Las redes sociales: una nueva herramienta de difusión. Recuperado de https://dialnet.unirioja.es/descarga/articulo/4796327.pdf

INEGI. (2016). Encuesta Nacional sobre Productividad y Competitividad de las micro, pequeñas y medianas empresas, (Enaproce). Recuperado de http://www.beta.inegi.org.mx/app/mapa/denue/\# 
EL USO DE REDES SOCIALES EN LAS MICROEMPRESAS PANIFICADORAS DE TABASCO.

Maldonado, G.; Ojeda, J.; Uc, L.; Valdez, L. y Medina, M. (2017). La Microempresa en México: un diagnóstico de su situación actual. Universidad Autónoma de Aguascalientes. ISBN 978-607-8523-06-1.

Marketing FCA. (2009). Recuperado de http://mktfcaunam.blogspot.com.co/

Martínez, G. (2012). Redes Sociales En: Temas de Informática para Enfermería. Cap. 16. La Habana: Ecimed.

Martos, A. (2010). Redes sociales. Madrid: Ediciones Anaya.

Mattileppaniemi. (2010). Targetin Young Voters in a Political Campaing in the 2007 Finnish General Election. Journal of Nonprofit \& Public Sector Marketing.

Mead, C. \& Liedholm, C. (1998). The dynamics of micro and small enterprises in development countries. World Development.

Medina, A. y Montjoy, R. (2015). El marketing directo en las redes sociales y su influencia en el campo empresarial. (Tesis de pregrado). Universidad Técnica de Machala, Machala, Ecuador.

Mejía, J. (2013). La Guía del Community Manager. Madrid: Ediciones Anaya.

Muñoz C.; Pérez, B. y Navarrete M. (2016). Las redes sociales y el desarrollo de las MiPyMES. Recuperado de http://132.248.9.34/hevila/CienciadesdeelOccidente/20152016/vol3/no1/7.pdf

Observatorio Regional de la Sociedad de la Información de la Junta de Castilla y León ORSI. (2012). Recuperado el 20 de 05 de 2015, de https://www.cyldigital.es/sites/default/files/library/guiamarketingdigital.pdf

Peng, Y. y Trappey, C. (2005). Internet and e-commerce adoption by the Taiwan semiconductor industry. Industrial Management \& Data Systems, 105(4), 476-490.

Poblete, O. (2013). El marketing digital y su importancia. Recuperado de http://blogs.infobae.com/teletrabajo/2013/06/26/el-marketingdigital-y-su-importancia/

Priego, J. (2013). Algunas consideraciones sobre la administración de las pequeñas empresas panaderas familiares en la ciudad de Villahermosa, Tabasco. México: UJAT. 
EL USO DE REDES SOCIALES EN LAS MICROEMPRESAS PANIFICADORAS DE TABASCO.

Ramírez, L. (2004). Cibermarketing: Una oportunidad de desarrollo dentro del sistema económico global para las empresas mexicanas. Razón y palabra.

Razón y Palabra. (2010). Razón y palabra. Recuperado el 20 de 05 de 2015 de: http://www.razonypalabra.org.mx/N/N71/NARIA/29\%20JODAR_REVISADO.pdf

Rotondaro, R. (2002). Defining the customer's expectations in e-business. Industrial Management \& Data Systems, 102(9), 476.

Sánchez, C. y Valdés, P. (2011). Efectividad instituciones de los programas de apoyo a las MIPyMES en México. Revista Internacional Administración y Finanzas.

Santes, R.; Navarrete, M. y García, C. (2017). Marketing digital para los consumidores del siglo XXI. Revista Hitos de Ciencias Económico Administrativas, 23(65), 24-37.

Santos M. y Guzmán L. (2017). Las microempresas y la reducción de la pobreza en Jalisco, México. Estudios regionales en economía, población y desarrollo. Cuadernos de Trabajo de la UACJ, Universidad Autónoma de Ciudad Juárez.

Smith, R. \& Chaffey, D. (2005). E-marketing excellence: at the heart of e-business. Oxford, UK: Butterworth Heinemann.

Soliman, F. \& Youssef, M. (2003). Internet-based e-commerce and its impact on manufacturing and business operations. Industrial Management \& Data Systems, 103(8), 546-552.

Treviño-Rodríguez, N. (2009). From a family-owned to a family-controlled business. Journal of Management History.

Trujillo, P. (2009). Caracterización Comercial De La Panadería Tradicional De La Ciudad De Manizales. Recuperado de http://ridum.umanizales.edu.co:8080/xmlui/bitstream/handle/6789/233/L\%C3\%B3pez_Tr ujillo_Pablo_Andres_2009.pdf?sequence=2

Williams, D., Crittenden, V., Keo, T. \& McCarty, P. (2012). The use of social media: An exploratory study of usage among digital natives. Journal of Public Affairs, 127-136.

Xiang, Z. \& Gretzel, U. (2010). Role of social media in online travel information search. Tourism Management, (31) 179-188. 